\title{
Quaternized chitosan promotes the antiproliferative effect of vemurafenib in melanoma cells by increasing cell permeability
}

This article was published in the following Dove Press journal: OncoTargets and Therapy

Min $\mathrm{Li}^{1}$

Ying Yang ${ }^{2,3}$

'Department of Oncology, Changsha Central Hospital, Changsha 4I0006, Hunan, PR China; ${ }^{2}$ Department of Plastic Surgery, Xiangya Hospital, Central South University, Changsha 4I0008, PR China; ${ }^{3}$ State Key Laboratory of Powder Metallurgy, Research Institute of Powder Metallurgy, Central South University, Changsha 4I0083, PR China
Correspondence: Ying Yang

Department of Plastic Surgery, Xiangya Hospital, Central South University, 87 Xiangya Road, Changsha 410008, PR China

Tel +8673189753014

Fax +8673184327332

Email forever_yang@csu.edu.cn
Background: As one of the most invasive cutaneous carcinomas among all types of skin cancer, malignant melanoma remains a severe challenge in oncology and plastic surgery. Selective smallmolecule inhibitors of V600E-mutant B-Raf (vemurafenib, for instance) have demonstrated satisfactory therapeutic efficacy in melanoma patients. However, acquired resistance during the period of drug administration has limited their clinical application.

Materials and methods: In the present study, human melanoma cells with the B-Raf ${ }^{\mathrm{V} 600 \mathrm{E}}$ mutation were treated with the indicated concentrations of vemurafenib, quaternized chitosan, or a combination of vemurafenib and quaternized chitosan. Cell proliferation and viability were evaluated using cell counting kit- 8 assay and DAPI staining, and the $\mathrm{IC}_{50}$ values for vemurafenib in melanoma cells were also determined. Cell apoptosis was evaluated by Live/Dead cell staining using confocal laser scanning microscopy and Annexin V-FITC Apoptosis detection using flow cytometry, respectively. The leakage of ATP and $\mathrm{K}^{+}$into the cell supernatants was measured to evaluate cell permeability. Furthermore, the surface charge variation of melanoma cells after drug treatment was determined by measuring the zeta potential of the cell membrane to clarify the electrostatic interaction between quaternized chitosan and the cells.

Results: Our results indicated that the addition of quaternized chitosan could promote the antiproliferative effect of vemurafenib in melanoma cells and could also promote the cell apoptosis of melanoma cells treated with vemurafenib. In addition, quaternized chitosan could increase cell permeability at early stages of co-culture, thus contributing to the improvement in intracellular drug uptake. Meanwhile, the majority of the negative surface charge of the cells was counteracted by the quaternized chitosan, indicating that the surface charge of melanoma cells was disturbed after the addition of quaternized chitosan.

Conclusion: This study indicated that disturbance of the surface charge of the cell membrane by quaternized chitosan is an important mechanism involved in changes in cell permeability, which promote the antiproliferative effect of vemurafenib in melanoma cells. Our preliminarily investigation provides new insights into the improvement of clinical melanoma therapy in the future.

Keywords: melanoma, vemurafenib, quaternized chitosan, antiproliferative, cell permeability

\section{Introduction}

Melanoma is one of the most invasive cutaneous carcinomas that is commonly seen in oncology and plastic surgery departments, and it accounts for $>70 \%$ of the deaths resulting from skin carcinoma annually. ${ }^{1}$ It has been reported that B-Raf mutations to glutamic acid (V600E) are found in nearly half of cutaneous melanomas. ${ }^{1,2}$ Selective small molecule inhibitors of V600E-mutant B-Raf, including vemurafenib, have 
demonstrated successively promoted clinical responses and survival rates compared with conventional chemotherapy in melanoma patients with B-Raf ${ }^{\mathrm{V} 600 \mathrm{E}}$ mutations. ${ }^{3,4}$ However, the median duration of the responses to B-Raf inhibitors in those clinical trials has been reported to be only $\sim 6$ months, ${ }^{3}$ which is due to the development of acquired resistance during the period of drug administration. ${ }^{5}$ Therefore, therapeutic strategies aimed at promoting early treatment efficacy and avoiding or delaying resistance are of great significance for kinase inhibitor therapy in melanomas.

As a widely used antibacterial agent in personal use and medical treatment, triclosan has demonstrated significant advantages over antibiotics due to its low risk of drug resistance and enhanced inhibition of biofilm formation. ${ }^{6-8}$ Furthermore, triclosan-coated polyglactin sutures have emerged as an option for decreasing the occurrence of surgical site infections in surgical operations. ${ }^{9,10}$ However, the potential of triclosan to induce tissue toxicity, endocrine disorders, and to promote tumor growth raised great concerns regarding its biological safety. ${ }^{11-13} \mathrm{Wu}$ et al reported that triclosan promoted sorafenib resistance in hepatocellular carcinoma cells because it induced the expression of drug resistance genes, accelerated clearance, and weakened antiproliferative effects of sorafenib. ${ }^{14}$ The topic of this study is of great importance in relation to the cautious use of triclosan-containing medical products in cancer patients in the future. Inspired by this previous research, we performed some experiments designed to reveal the relationship between the non-antibiotic antimicrobial agents and tumor cells.

We have conducted a series of investigations on the antibacterial properties of quaternized chitosan, a non-antibiotic antimicrobial agent similar to triclosan. As a biodegradable nontoxic biopolymer derived from chitosan, quaternized chitosan exhibits satisfactory antimicrobial activity and biocompatibility both in vitro and in vivo, as elaborately described in our previous studies. ${ }^{15-17}$ Moreover, we also found that quaternized chitosan-coated sutures showed comparable anti-infection potential and cytocompatibility with triclosan-coated sutures. ${ }^{18}$ It has been verified that quaternized chitosan with positively charged quaternary ammonium groups exerts broad-spectrum antimicrobial effects via electrostatic interactions with microbes with negatively charged phosphoryl groups, thus affecting the cytoplasmic membrane integrity. ${ }^{19}$ Consequently, we wondered whether such an electrostatic interaction exists between quaternized chitosan and melanoma cells, which might facilitate the therapeutic effect of vemurafenib at the early stage.

\section{Materials and methods Cell culture and reagents}

A375 human melanoma cells with the B-Raf ${ }^{\mathrm{V} 600 \mathrm{E}}$ mutation were purchased from the Type Culture Collection of the Chinese Academy of Sciences (Shanghai, PR China). Cells were grown in an incubator at $37^{\circ} \mathrm{C}$ and $5 \% \mathrm{CO}_{2}$. Cells were grown in DMEM (Gibco, Thermo Fisher Scientific, Waltham, MA, USA) supplemented with 10\% FBS, 2 mM L-glutamine, $100 \mathrm{UI} / \mathrm{mL}$ streptomycin, and $100 \mathrm{UI} / \mathrm{mL}$ penicillin (SigmaAldrich Co., St Louis, MO, USA). Vemurafenib, a known inhibitor of B-Raf ${ }^{\mathrm{V} 600 \mathrm{E}}$, was purchased from Selleck Chemical ([PLX4032] Houston, TX, USA). Quaternized chitosan was prepared by combining glycidyl trimethylammonium chloride and chitosan as previously reported. ${ }^{15-18}$ A $10 \mathrm{mM}$ vemurafenib solution was produced by dissolving the chemical in $1 \mathrm{~mL}$ DMSO (Sigma-Aldrich Co.).

\section{Cell proliferation and viability assays}

Human melanoma cells at a concentration of $1 \times 10^{4}$ cells/well were seeded in 96-well plates and cultured for 24 hours prior to drug treatment. In our study, cells were treated with vemurafenib $(2 \mu \mathrm{M})$, quaternized chitosan $(100 \mu \mathrm{g} / \mathrm{mL})$, or a mixture of vemurafenib $(2 \mu \mathrm{M})$ and quaternized chitosan $(100 \mu \mathrm{g} / \mathrm{mL})$. Wells containing only culture medium served as blank controls. The cell viability in each group was examined at $0,24,48,72$, and 96 hours after co-culture using a cell counting kit-8 (CCK-8) assay. ${ }^{15,16}$ At each time point, a total of $10 \mu \mathrm{L}$ of CCK-8 solution (Dojindo Molecular Technologies, Kumamoto, Japan) was added into each well, and the plates were then incubated for $\sim 4$ hours. Then, the absorbance of the wells in each group was determined by a Synergy HT microplate reader (Bio-Tek Instruments, Winooski, VT, USA) at a wavelength of $450 \mathrm{~nm}$.

To ascertain the influence of quaternized chitosan on the $\mathrm{IC}_{50}$ values of melanoma cells during the administration of vemurafenib, melanoma cells were treated with vemurafenib at concentrations varying from $0.01 \mu \mathrm{M}$ to $10 \mu \mathrm{M}$, together with quaternized chitosan at a concentration of $100 \mu \mathrm{g} / \mathrm{mL}$. The condition leading to a $50 \%$ inhibition of viability was defined as the $\mathrm{IC}_{50}$ for the drug. In addition, cells in each well were fixed with $4 \%$ neutral formaldehyde buffer solution for $\sim 30$ minutes at 48 and 96 hours after incubation, and then permeabilized with $0.1 \%$ Triton X-100 in PBS for 15 minutes. Subsequently, the cell nuclei were stained with DAPI for 15 minutes and then visualized by confocal laser scanning microscopy ([CLSM] Leica TCS SP8, Leica Microsystems, Wetzlar, Germany). 


\section{Cell apoptosis assays}

Firstly, cell apoptosis in different groups was evaluated using a Live/Dead Cell kit (Abcam, Cambridge, UK) as described in our previous study. ${ }^{15}$ Melanoma cells at a concentration of $5 \times 10^{5}$ cells/well were seeded in 12-well plates and cultured for 48 hours. After being washed with PBS, cells were stained with $300 \mu \mathrm{L}$ of mixture dye for 15 minutes and were then scanned by CLSM (Leica TCS SP8). Live cells with esterase activity appeared green, whereas dead cells with damaged membranes appeared red, as described in the manufacturer's protocol.

Secondly, the effects of different treatments on cell apoptosis were further analyzed on a BD LSRFortessa flow cytometer (BD Biosciences, San Jose, CA, USA), as described in a previously reported method. ${ }^{20}$ Melanoma cells were incubated with the indicated treatments for 48 hours as described previously. Then, cells were washed twice with PBS after trypsinization. Collected cells were suspended in $400 \mu \mathrm{L} 1 \times$ binding buffer, then $5 \mu \mathrm{L}$ of Annexin V-FITC (BD Biosciences) was added to the cell suspension, and incubated for 30 minutes on ice in a dark environment. Then, $5 \mu \mathrm{L}$ propidium iodide (BD Biosciences) was added and incubated for 5 minutes. After being washed with PBS, cells were resuspended in $100 \mu \mathrm{L}$ binding buffer for the flow cytometry analysis. The final results were analyzed by FlowJo software (TreeStar, Ashland, OR, USA).

\section{Cell permeability assays}

The integrity and permeability of the cell membrane were evaluated at $0,6,12$, and 24 hours after drug administration using the ATP and $\mathrm{K}^{+}$leakage assay. In brief, $100 \mu \mathrm{L}$ samples were collected from the wells at the desired time points as mentioned previously, and then centrifuged at $500 \mathrm{rpm}$ for 5 minutes. The cell-free supernatant was processed using a CellTiter-Glo ${ }^{\mathrm{TM}}$ luminescent cell viability assay kit (Promega Corporation, Fitchburg, WI, USA), and then analyzed using a luminometer (Infinite M200 PRO, TECAN, Mannedorf, Switzerland). Furthermore, the concentration of $\mathrm{K}^{+}$in the collected supernatant was measured by inductively coupled plasma-optical emission spectroscopy (Optima 7000DV, PerkinElmer Inc., Waltham, MA, USA).

\section{Measurement of surface charge variation among melanoma cells}

To clarify whether changes in cell permeability resulted from the disturbance of surface charge in melanoma cells, we measured the zeta potential, which is representative of the surface charge of the melanoma cells, before and after drug treatment, according to a modified procedure. ${ }^{21}$ In brief, after co-culture with drugs as described previously, cells were collected and resuspended in ultrapure water, and the measurement time points in each group were 12 and 24 hours. At each desired time point, the samples were exposed to ultrasound $(20 \mathrm{~Hz})$ for 2 minutes to obtain a homogeneous colloidal suspension, and then examined by a Zetasizer Nano instrument (Malvern Panalytical Ltd, Worcestershire, UK). Additionally, the zeta potential of the cells prior to the drug intervention was also measured.

\section{Statistical analysis}

SPSS software 19.0 (IBM Corporation, Armonk, NY, USA) was used to perform the statistical analyses. Quantitative data are presented as the mean $\pm \mathrm{SD}$. All in vitro cell experiments in the present investigation were repeated three times. The $\mathrm{IC}_{50}$ values for vemurafenib in melanoma cells were calculated using GraphPad Prism 5 for Windows (GraphPad Software, Inc., La Jolla, CA, USA). One-way analysis and a nonparametric test were used to determine the level of significance. $P<0.05$ represented a significant difference.

\section{Results and discussion}

In this study, we investigated the influences of quaternized chitosan on the antiproliferative effects of vemurafenib in melanoma cells by evaluating cell proliferation, viability, apoptosis, permeability, and surface charge. We hypothesized that combined treatment with vemurafenib and quaternized chitosan could offer new insights into the improvement of clinical melanoma therapy in the future.

As shown in Figure 1A, the proliferation of cells treated with different interventions varied among the four groups from 48 to 96 hours of co-culture. The cells in groups V (vemurafenib) and V + QC (vemurafenib + quaternized chitosan) exhibited significantly less proliferation than did those in the other two groups at 48, 72, and 96 hours of culture $(P<0.01)$; furthermore, the cell proliferation in the QC group was slightly lower than that in the blank control group at 48 and 72 hours $(P<0.05)$. The cell numbers in the $\mathrm{V}+\mathrm{QC}$ group were lower than those in the $\mathrm{V}$ group from 48 to 96 hours $(P<0.05)$. Meanwhile, the $\mathrm{IC}_{50}$ of vemurafenib in melanoma cells decreased with the combined utilization of quaternized chitosan, as shown in Figure 1B $(P<0.05)$. In addition, the number of cells stained with DAPI in the four groups after 48 and 96 hours of incubation is displayed in Figure 1C, and these numbers were consistent with the cell proliferation curves shown in Figure 1A. The cell proliferation and viability assessment results indicated that the 

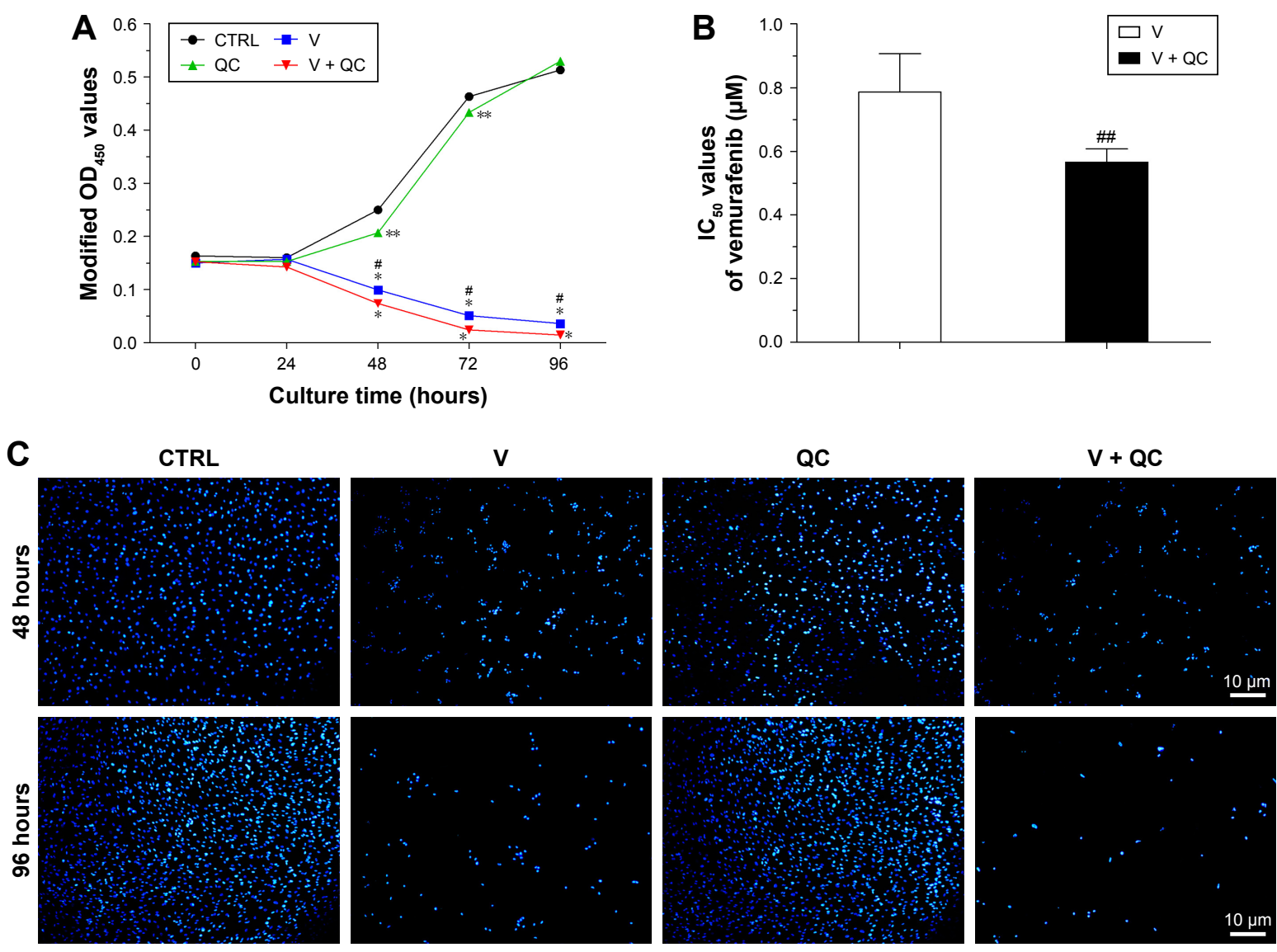

Figure I Quaternized chitosan promoted the in vitro antiproliferative effect of vemurafenib in melanoma cells.

Notes: Melanoma cells were treated with medium control, vemurafenib, quaternized chitosan, or a mixture of vemurafenib and quaternized chitosan. (A) The proliferation

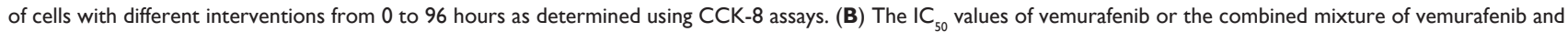
quaternized chitosan in melanoma cells. (C) Representative images of cells stained with DAPI after 48 and 96 hours of culture. The scale bar is $10 \mu \mathrm{m}$ as indicated in the last image of each row. ${ }^{*} P<0.01$, compared with the CTRL and QC groups at the corresponding time points. $* * P<0.05$, compared with the CTRL group at the corresponding time points. ${ }^{\#} P<0.05$, compared with the $V+Q C$ group at the corresponding time points. ${ }^{\#} P<0.05$, compared with the $V$ group.

Abbreviations: CCK-8, cell counting kit-8; CTRL, blank control; QC, quaternized chitosan; $\mathrm{V}$, vemurafenib.

addition of quaternized chitosan could promote the antiproliferative effect of vemurafenib in melanoma cells, which preliminarily validated our hypothesis.

Meanwhile, we also evaluated the cell apoptosis in different groups through Live/Dead Cell assay on CLSM and Annexin V-FITC Apoptosis Detection assay using flow cytometry, respectively. As shown in Figure 2A, the human melanoma cells in the $\mathrm{V}$ and $\mathrm{V}+\mathrm{QC}$ groups displayed a significantly increased number of dead cells compared with the control and QC groups. In addition, more red fluorescence representative of dead cells was found in the V + QC group than in the $\mathrm{V}$ group. Moreover, we performed flow cytometry to further confirm what was found in the CLSM observation. As demonstrated in Figure 2B and C, the proportion of apoptotic cells increased markedly in the $\mathrm{V}$ and $\mathrm{V}+\mathrm{QC}$ groups compared to the control and $\mathrm{QC}$ groups $(P<0.01)$. In addition, the percentage of apoptotic cells in the $\mathrm{V}+\mathrm{QC}$ group was also higher than those in the $\mathrm{V}$ group $(P<0.05)$. Therefore, the addition of quaternized chitosan could promote the cell apoptosis of melanoma cells treated with vemurafenib, thus facilitating the therapeutic efficacy of vemurafenib in suppressing the rapid growth of melanoma cells.

To clarify the effects of quaternized chitosan on the permeability of melanoma cells, we measured the levels of ATP and $\mathrm{K}^{+}$in the cell supernatant after drug treatment. We found that there was a significant increase in the leakage of ATP and $\mathrm{K}^{+}$ from 6 to 24 hours of co-culture in the QC and V + QC groups compared with the $\mathrm{V}$ and control groups, as shown in Figure 3 $(P<0.01)$. The leakage of ATP after 24 hours of culture and of $\mathrm{K}^{+}$after 12 and 24 hours of culture were both higher in the $\mathrm{V}+\mathrm{QC}$ group than that in the QC group $(P<0.05)$. The leakage of ATP and $\mathrm{K}^{+}$at 24 hours in the $\mathrm{V}$ group was also higher than that in the blank control group $(P<0.05)$. It seemed that the addition of quaternized chitosan could increase the cell permeability at the early stages of co-culture. This effect contributed to the improvement in intracellular drug uptake, 

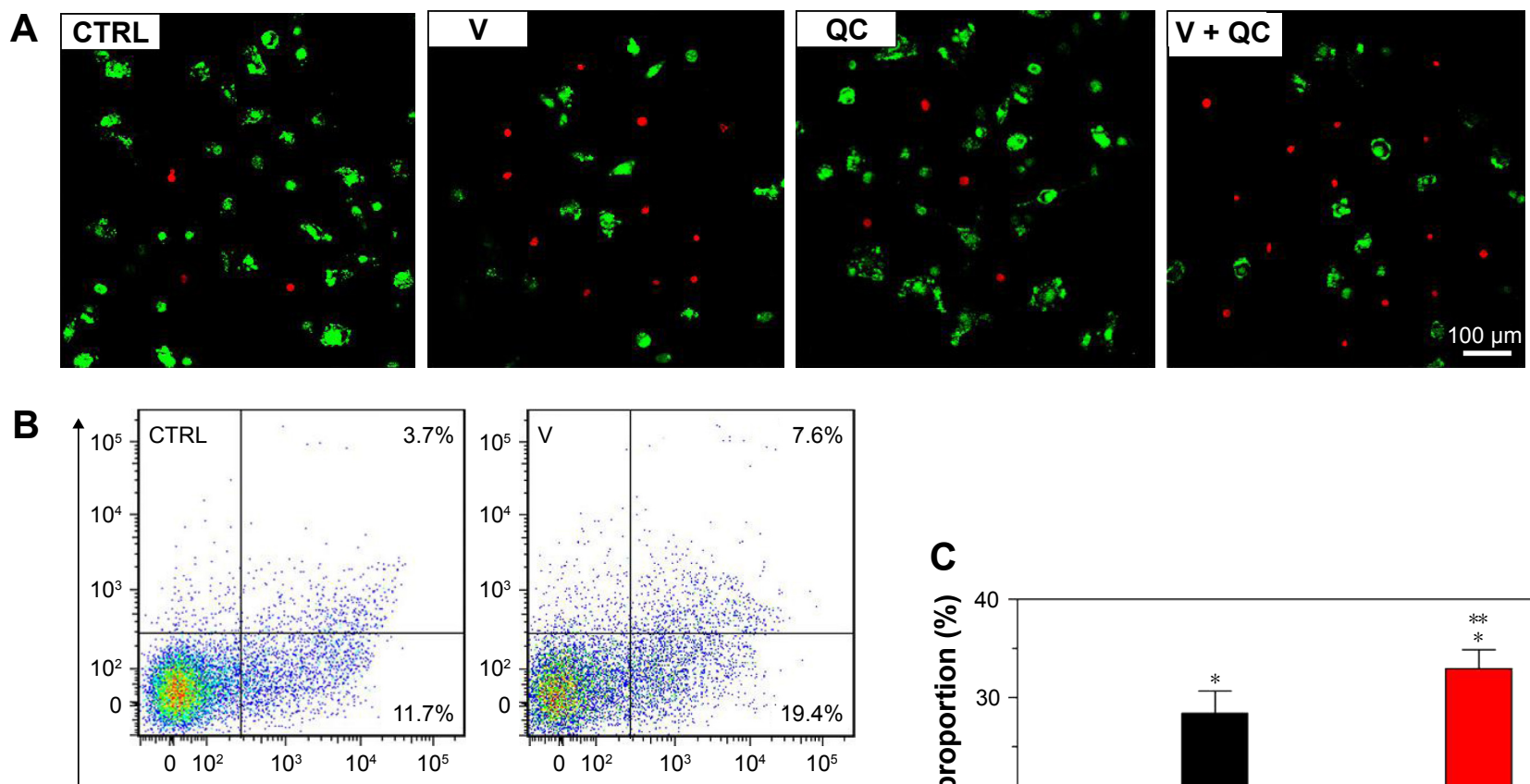

$\bar{\alpha}$
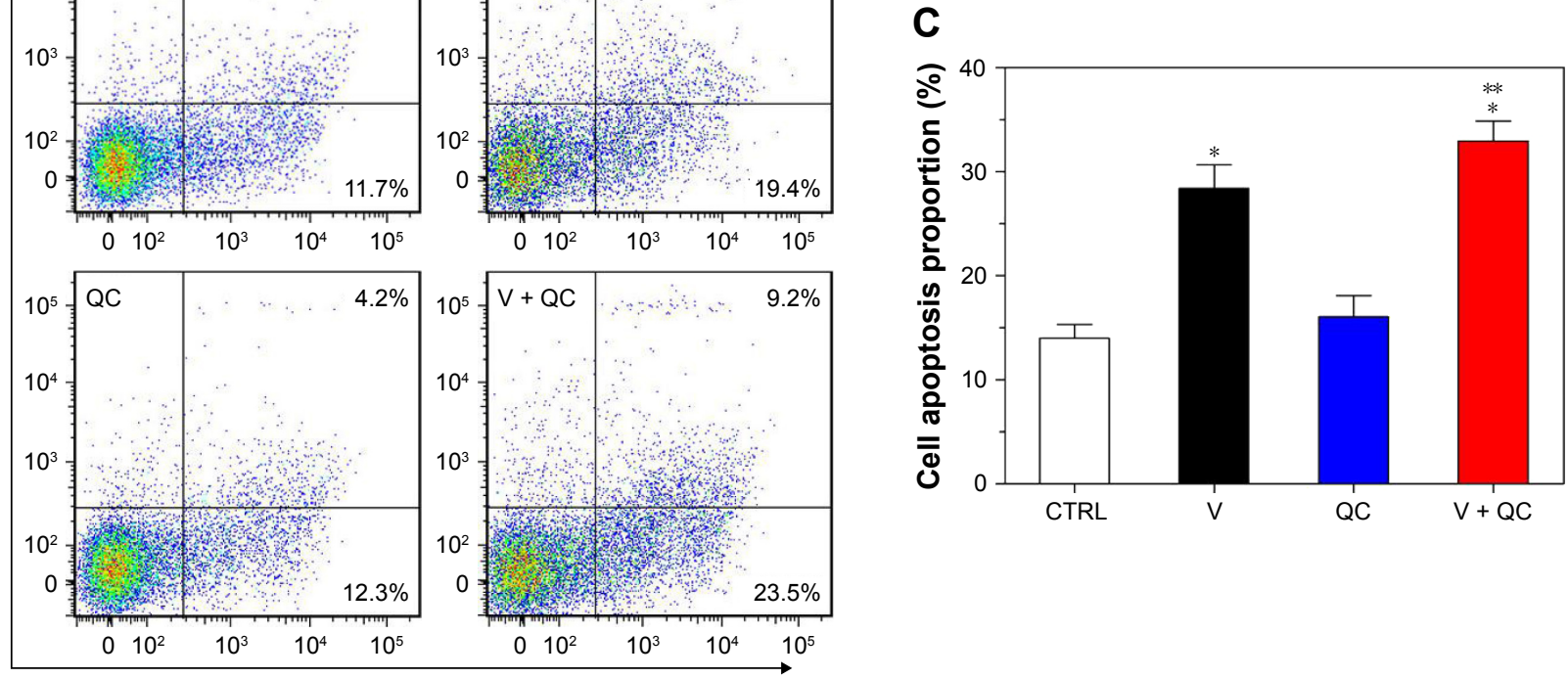

Annexin V-FITC

Figure 2 Quaternized chitosan showed a synergetic effect on increasing cell apoptosis caused by vemurafenib in melanoma cells.

Notes: Melanoma cells were treated with medium control, vemurafenib, quaternized chitosan, or a mixture of vemurafenib and quaternized chitosan. (A) Cell viability observed using the Live/Dead Cell kit after a 48-hour incubation. Live cells with esterase activity appeared green, whereas dead cells with damaged membranes appeared red. (B) Cell apoptosis evaluated by flow cytometry after being stained with the Annexin-V and PI after a 48-hour incubation. (C) Quantification of the ratio of apoptotic cells in different groups after a 48 -hour incubation. The scale bar is $100 \mu \mathrm{m}$ as indicated in the last image of each row. $* P<0.01$, compared with the $C T R L$ and $Q C$ groups. $* * P<0.05$, compared with the $V$ group.

Abbreviations: CTRL, blank control; PI, propidium iodide; QC, quaternized chitosan; V, vemurafenib.

thus enhancing the chemotherapeutic efficacy of vemurafenib in melanoma cells. Although quaternized chitosan increased the permeability of the cell membrane, it was found that quaternized chitosan itself exhibited no antiproliferative effect on melanoma cells at the end of the co-culture.

Furthermore, by measuring the zeta potential of the cell membrane, we found that the surface charge of the melanoma cells was typically negative $(-18.2 \pm 0.9 \mathrm{mV})$, as shown in Figure 4, and the absolute zeta potential value of the cells treated with quaternized chitosan alone and combination of vemurafenib and quaternized chitosan was significantly decreased after 12 and 24 hours of co-culture when compared to cells treated with only vemurafenib and to cells without drug treatment $(P<0.01)$. Moreover, the absolute zeta potential value of cells treated with quaternized chi- tosan (groups QC and V + QC) both exhibited a declining trend from 12 to 24 hours of co-culture $(P<0.05)$, while the absolute zeta potential value of the cells treated with only vemurafenib was relatively stable and similar to that of cells without intervention $(P>0.05)$. Therefore, most of the negative surface charge of the cells was counteracted by the quaternized chitosan via its positively charged quaternary ammonium groups. It has been verified that the acidification of the tumor microenvironment caused by the deregulation of cancer cell metabolism is one important characteristic of malignant melanoma, ${ }^{22,23}$ and the chemotherapeutic efficacy of vemurafenib in melanoma cells may be enhanced by the partial neutralization of the tumor microenvironment mediated by the positively charged quaternized chitosan. The improvement in the tumor microenvironment could 

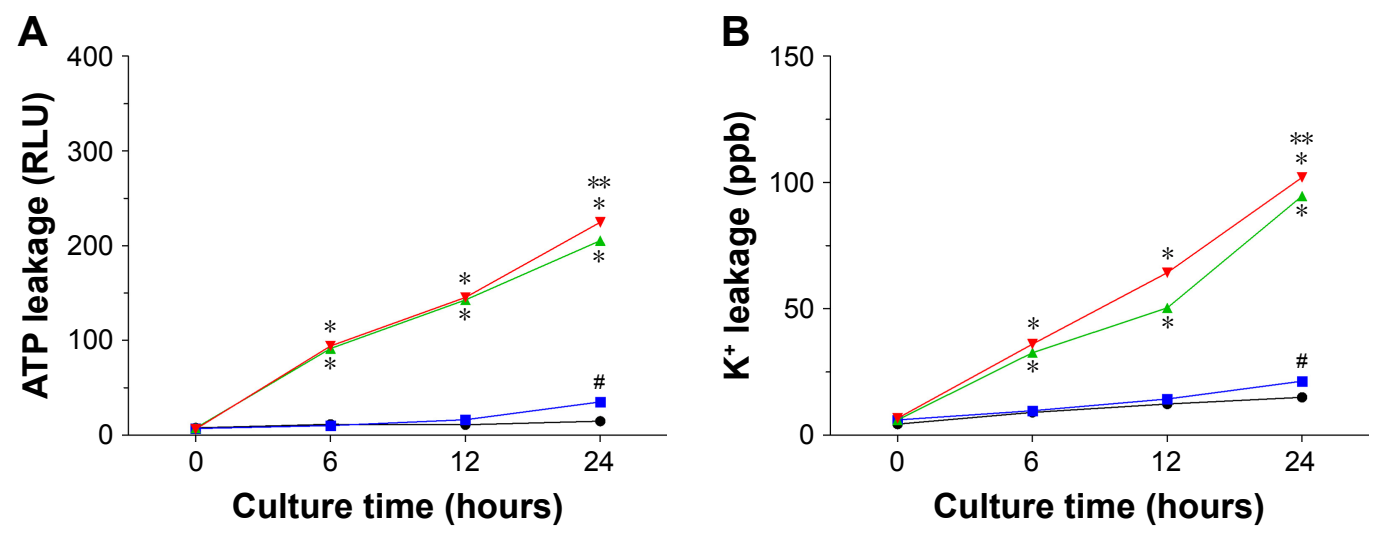

$$
\rightarrow \mathrm{CTRL} \rightarrow \mathrm{V} \rightarrow \mathrm{QC} \rightarrow \mathrm{V}+\mathrm{QC}
$$

Figure 3 Quaternized chitosan increased the permeability of melanoma cells.

Notes: Melanoma cells were treated with medium control, vemurafenib, quaternized chitosan, or a mixture of vemurafenib and quaternized chitosan. The leakage curves of intracellular (A) ATP and (B) $\mathrm{K}^{+}$from cells exposed to different interventions from 0 to 24 hours. $* P<0.01$, compared with the CTRL and $V$ groups at the corresponding time points. ${ }^{* * P}<0.05$, compared with the $\mathrm{Q} C$ group at the corresponding time points. ${ }^{*} P<0.05$, compared with the $C T R L$ group at the corresponding time points.

Abbreviations: CTRL, blank control; QC, quaternized chitosan; V, vemurafenib.

be advantageous for decreasing the risk of drug resistance, thus promoting the antitumor efficacy of kinase inhibitors in melanoma cells.

\section{Conclusion}

In general, we conclude that the disturbance of the cell membrane surface charge induced by quaternized chitosan is one of the important mechanisms involved in the changes in cell permeability at the early stage after drug administration, and this effect ultimately enhances the antiproliferative effect of vemurafenib in melanoma cells. However, direct

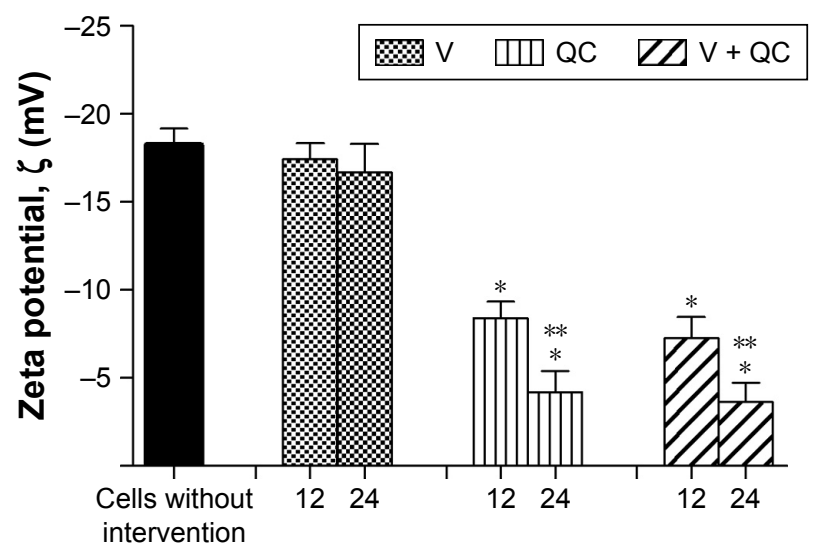

Time points for drug intervention (hours)

Figure 4 Quaternized chitosan disturbed the surface charge of melanoma cells via electrostatic interaction.

Notes: Melanoma cells were treated with medium control, vemurafenib, quaternized chitosan, or a combination of vemurafenib and quaternized chitosan. The zeta potentials of the cells with or without drug interventions were measured after they were homogeneously suspended in ultrapure water. $* P<0.01$, compared with the CTRL and $V$ (including 12 and 24 hours) groups. $* * P<0.05$, compared with 12 hours after culture within the $\mathrm{QC}$ and $\mathrm{V}+\mathrm{QC}$ groups.

Abbreviations: CTRL, blank control; QC, quaternized chitosan; $\mathrm{V}$, vemurafenib. evidence for increase in intracellular vemurafenib uptake due to this elevated cell permeability and other mechanisms remain to be explored in our future investigations. In view of the improvement in antiproliferative efficacy of a kinase inhibitor in melanoma cells in the present study, and because of its satisfactory antimicrobial performances reported in our previous studies, ${ }^{16-18}$ quaternized chitosan-coated sutures could be a recommended alternative for wound closure after radical excision of malignant melanoma.

\section{Acknowledgments}

This work was supported by the National Natural Science Foundation for Youths (grant number: 81802136), the Young Researcher Fund of Xiangya Hospital, Central South University (grant number: 2017Q18), and the Initial Postdoctoral Research Fund of Central South University (serial number: 205432).

\section{Author contributions}

We confirm that all authors (ML and YY) of the present study made substantial contributions to the experimental design, collection, analysis, and illustration of data. Both authors contributed to data analysis, drafting and revising the article, gave final approval of the version to be published, and agree to be accountable for all aspects of the work.

\section{Disclosure}

The authors report no conflicts of interest in this work.

\section{References}

1. Bucheit AD, Davies MA. Emerging insights into resistance to BRAF inhibitors in melanoma. Biochem Pharmacol. 2014;87(3):381-389. 
2. Davies H, Bignell GR, Cox C, et al. Mutations of the BRAF gene in human cancer. Nature. 2002;417(6892):949-954.

3. Chapman PB, Hauschild A, Robert C, et al. Improved survival with vemurafenib in melanoma with BRAF V600E mutation. N Engl J Med. 2011;364(26):2507-2516.

4. McArthur GA, Chapman PB, Robert C, et al. Safety and efficacy of vemurafenib in $\mathrm{BRAF}^{\mathrm{V} 600 \mathrm{E}}$ and $\mathrm{BRAF}^{\mathrm{V} 600 \mathrm{~K}}$ mutation-positive melanoma (BRIM-3): extended follow-up of a phase 3, randomised, open-label study. Lancet Oncol. 2014;15(3):323-332.

5. Nazarian R, Shi H, Wang Q, et al. Melanomas acquire resistance to B-RAF(V600E) inhibition by RTK or N-RAS upregulation. Nature. 2010;468(7326):973-977.

6. Goodman M, Naiman DQ, Lakind JS. Systematic review of the literature on triclosan and health outcomes in humans. Crit Rev Toxicol. 2018;48(1):1-51.

7. Bhende $S$, Burkley D, Nawrocki J. In vivo and in vitro anti-bacterial efficacy of absorbable barbed polydioxanone monofilament tissue control device with triclosan. Surg Infect. 2018;19(4):430-437.

8. Maiden MM, Hunt AMA, Zachos MP, et al. Triclosan is an aminoglycoside adjuvant for eradication of Pseudomonas aeruginosa biofilms. Antimicrob Agents Chemother. 2018;62(6):e00146-18.

9. Nakamura T, Kashimura N, Noji T, et al. Triclosan-coated sutures reduce the incidence of wound infections and the costs after colorectal surgery: a randomized controlled trial. Surgery. 2013;153(4):576-583.

10. Laas E, Poilroux C, Bézu C, et al. Antibacterial-coated suture in reducing surgical site infection in breast surgery: a prospective study. Int $J$ Breast Cancer. 2012;2012:819578-8.

11. Wang $X$, Chen $X$, Feng $X$, et al. Triclosan causes spontaneous abortion accompanied by decline of estrogen sulfotransferase activity in humans and mice. Sci Rep. 2015;5:18252.

12. Weatherly LM, Gosse JA. Triclosan exposure, transformation, and human health effects. J Toxicol Environ Health B Crit Rev. 2017;20(8): 447-469.

13. Yueh MF, Taniguchi $\mathrm{K}$, Chen $\mathrm{S}$, et al. The commonly used antimicrobial additive triclosan is a liver tumor promoter. Proc Natl Acad Sci US A. 2014;111(48):17200-17205.
14. Wu M, Zhao G, Zhuang X, et al. Triclosan treatment decreased the antitumor effect of sorafenib on hepatocellular carcinoma cells. Onco Targets Ther. 2018;11:2945-2954.

15. Yang Y, Ao H, Wang Y, et al. Cytocompatibility with osteogenic cells and enhanced in vivo anti-infection potential of quaternized chitosanloaded titania nanotubes. Bone Res. 2016;4:16027.

16. Yang Y, Yang S, Wang Y, et al. Anti-infective efficacy, cytocompatibility and biocompatibility of a 3D-printed osteoconductive composite scaffold functionalized with quaternized chitosan. Acta Biomater. 2016;46:112-128.

17. Yang Y, Chu L, Yang S, et al. Dual-functional 3D-printed composite scaffold for inhibiting bacterial infection and promoting bone regeneration in infected bone defect models. Acta Biomater. 2018;79:265-275.

18. Yang Y, Yang SB, Wang YG, Zhang SH, Yu ZF, Tang TT. Bacterial inhibition potential of quaternised chitosan-coated VICRYL absorbable suture: an in vitro and in vivo study. J Orthop Translat. 2017;8: 49-61.

19. Marcotte L, Barbeau J, Lafleur M. Permeability and thermodynamics study of quaternary ammonium surfactants-phosphocholine vesicle system. J Colloid Interface Sci. 2005;292(1):219-227.

20. Ma H, Jin S, Yang W, et al. Interferon-alpha enhances the antitumour activity of EGFR-targeted therapies by upregulating RIG-I in head and neck squamous cell carcinoma. Br J Cancer. 2018;118(4):509-521.

21. Wilson WW, Wade MM, Holman SC, Champlin FR. Status of methods for assessing bacterial cell surface charge properties based on zeta potential measurements. J Microbiol Methods. 2001;43(3):153-164.

22. Böhme I, Bosserhoff AK. Acidic tumor microenvironment in human melanoma. Pigment Cell Melanoma Res. 2016;29(5):508-523.

23. Fischer GM, Vashisht Gopal YN, McQuade JL, Peng W, Deberardinis RJ, Davies MA. Metabolic strategies of melanoma cells: mechanisms, interactions with the tumor microenvironment, and therapeutic implications. Pigment Cell Melanoma Res. 2018;31(1):11-30.
OncoTargets and Therapy

\section{Publish your work in this journal}

OncoTargets and Therapy is an international, peer-reviewed, open access journal focusing on the pathological basis of all cancers, potential targets for therapy and treatment protocols employed to improve the management of cancer patients. The journal also focuses on the impact of management programs and new therapeutic agents and protocols on

\section{Dovepress}

patient perspectives such as quality of life, adherence and satisfaction. The manuscript management system is completely online and includes a very quick and fair peer-review system, which is all easy to use. Visit http://www.dovepress.com/testimonials.php to read real quotes from published authors. 